\title{
Sleeve resection after neoadjuvant chemoimmunotherapy in the treatment of locally advanced non-small cell lung cancer
}

\author{
Jie Dai ${ }^{1 \#}$, Xinsheng Zhu ${ }^{1 \#}$, Dianke $\mathrm{Li}^{1 \#}$, Yan Huang ${ }^{2}$, Xiaogang Liu ${ }^{1}$, Wenxin $\mathrm{He}^{1}$, Liang Duan ${ }^{1}$, \\ Deping Zhao ${ }^{1}$, Yuming Zhu ${ }^{1}$, Chang Chen ${ }^{1}$, Mariano Provencio ${ }^{3}$, Robert A. Ramirez ${ }^{4}$, Mara B. Antonoff ${ }^{5}$, \\ Chunyan $\mathrm{Wu}^{2}$, Gening Jiang ${ }^{1}$
}

${ }^{1}$ Department of Thoracic Surgery, Shanghai Pulmonary Hospital, Tongji University School of Medicine, Shanghai, China; ${ }^{2}$ Department of Pathology, Shanghai Pulmonary Hospital, Tongji University School of Medicine, Shanghai, China; ${ }^{3}$ Hospital Universitario Puerta de HierroMajadahonda, Madrid, Spain; ${ }^{4}$ Division of Hematology/Oncology, Vanderbilt University Medical Center, Nashville, TN, USA; ${ }^{5}$ Department of Thoracic and Cardiovascular Surgery, University of Texas MD Anderson Cancer Center, Houston, TX, USA

Contributions: (I) Conception and design: C Wu, G Jiang; (II) Administrative support: Y Zhu, C Chen, M Provencio, RA Ramirez, MB Antonoff; (III) Provision of study materials or patients: X Liu, W He, L Duan, D Zhao; (IV) Collection and assembly of data: J Dai, X Zhu, D Li, Y Huang; (V) Data analysis and interpretation: J Dai, X Zhu; (VI) Manuscript writing: All authors; (VII) Final approval of manuscript: All authors.

\#These authors contributed equally to this work.

Correspondence to: Gening Jiang, MD; Chunyan Wu, MD. No 507 Zhengmin Road, Shanghai 200433, China. Email: geningjiang@tongji.edu.cn; wuchunyan581@163.com.

Background: We aimed to characterize the outcomes of sleeve resection after neoadjuvant chemoimmunotherapy for the treatment of non-small cell lung cancer (NSCLC), including perioperative and oncologic outcomes, and to identify any impact of operative approach on resultant findings.

Methods: We identified patients with NSCLC who underwent sleeve resection after $\geq 2$ cycles of neoadjuvant chemoimmunotherapy between May 2019 and April 2021 and retrospectively reviewed clinical records. Perioperative data were collected and compared between video-assisted thoracoscopic surgery (VATS) ( $\mathrm{n}=8$ ) and thoracotomy ( $\mathrm{n}=15)$ groups. Immunohistochemistry (IHC) scores were compared between tumors with and without major pathological response (MPR).

Results: Twenty-three patients met inclusion criteria, with clinical stages as follows: IB, 2 (8.7\%); IIIA, 14 (60.9\%); and IIIB, 7 (30.4\%). Treatment-related adverse events (TRAE) were recorded in 17 patients (73.9\%), including anemia and neutropenia, with no patients exhibiting serious TRAE. Radiological evaluation revealed 5 (21.7\%) patients with complete response (CR), 14 (60.9\%) with partial response (PR), and 4 $(17.4 \%)$ with stable disease (SD). Complete resection was accomplished for all patients. One VATS procedure was converted to thoracotomy due to extensive pleural adhesions. There were no significant differences in intraoperative blood loss $(87.5 \pm 51.8$ vs. $193.9 \pm 145.3 \mathrm{~mL})$, operative time $(198.8 \pm 79.7$ vs. $225.5 \pm 55.0 \mathrm{~min})$, number of lymph node examined $(16.9 \pm 6.6$ vs. 18.2 \pm 6.5$)$, and hospital stay $(5.5 \pm 2.8 v s .9 .2 \pm 11.2$ days $)$ between the VATS and thoracotomy groups (all $\mathrm{P}>0.05$ ). Postoperative complications occurred in 3 patients, and 1 patient died of bronchopleural fistula (BPF) in the thoracotomy group. Complete pathological response (CPR) and MPR were achieved in seven (30.4\%) and 13 (56.5\%) patients, respectively. Both preoperative histopathology $(\mathrm{P}=0.024)$ and radiological response $(\mathrm{P}=0.002)$ were significantly associated with MPR. In postoperative specimens with MPR, the IHC scores of cluster of differentiation (CD)4, CD8, and CD20 were modestly higher, while programmed cell death receptor 1 (PD-1), lymphocyte-activation gene 3 (LAG3) and T cell immunoglobulin and ITIM domain (TIGIT) were lower compared with non-MPR specimens, albeit insignificantly.

Conclusions: Sleeve resection after neoadjuvant chemoimmunotherapy was feasible in patients with locally advanced NSCLC. Perioperative outcomes were comparable between the VATS and thoracotomy groups.

$\wedge$ ORCID: 0000-0002-5936-607X. 
Keywords: Neoadjuvant chemoimmunotherapy; sleeve resection; non-small cell lung cancer (NSCLC)

Submitted Oct 12, 2021. Accepted for publication Feb 21, 2022.

doi: $10.21037 /$ tlcr-22-56

View this article at: https://dx.doi.org/10.21037/tlcr-22-56

\section{Introduction}

The prognosis of locally advanced non-small cell lung cancer (NSCLC) remains poor, with 5-year survival of approximately $30 \%$ over the past few decades (1). Previous investigators have aimed to improve oncologic outcomes in this patient cohort, with multimodality therapies currently considered standard treatment $(2,3)$. However, the value of neoadjuvant chemotherapy is limited, with an increase of only $5 \%$ in 5 -year survival compared to surgery alone $(4,5)$. Recently, programmed cell death receptor 1 (PD-1) and programmed cell death ligand 1 (PD-L1) checkpoint inhibitors have shown promise in the treatment of locally advanced NSCLC $(6,7)$. Furthermore, adjuvant atezolizumab was shown to improve the disease-free survival in patients with resected NSCLC (8). Therefore, a neoadjuvant immunotherapy approach in this population is attractive.

For lung cancer invading the main bronchus, sleeve resection was recommended to avoid pneumonectomy and reserve more cardiac or pulmonary function, with promising perioperative and survival outcomes (9). However, the safety of sleeve resection after neoadjuvant immunotherapy has not yet been clearly elucidated, with concerns regarding potentially increased surgical risks, such as hemorrhage, persistent air leakage, bronchopleural fistula (BPF), related to both tissue adhesions and fibrosis as well as impact on the obligatory reconstruction of anatomic structures $(10,11)$.

This study aims to characterize perioperative outcomes of sleeve resection after neoadjuvant chemoimmunotherapy, as well as to identify any differences between operative approaches. In addition, we seek to investigate the oncologic outcomes of sleeve resections after induction chemoimmunotherapy and to explore potential predictors of pathological response in the treatment of NSCLC. We present the following article in accordance with the STROBE reporting checklist (available at https://tlcr. amegroups.com/article/view/10.21037/tlcr-22-56/rc).

\section{Methods}

\section{Study design and patient selection}

This study protocol was approved by the Ethics Committee of Shanghai Pulmonary Hospital (No. 19216XW-4), and the study protocols conformed to the provisions of the Declaration of Helsinki (as revised in 2013). For its retrospective nature, written consents from the included patients were not needed. The clinical data of patients who underwent sleeve resection after neoadjuvant chemoimmunotherapy between May 2019 and April 2021 at Shanghai Pulmonary Hospital were retrospectively collected. The primary inclusion criteria in clinical trial were as follows: (I) age 18-75 years; (II) Eastern Cooperative Oncology Group (ECOG) score 0-1; (III) clinical stage II-IIIB [N2]; (IV) epidermal growth factor receptor (EGFR) and anaplastic lymphoma kinase (ALK) wild-type; and (V) anticipation of complete resectability (R0 resection) after neoadjuvant therapy. The exclusion criteria of the study were as follows: (I) patients with concurrent additional malignancies; (II) patients that had undergone any other neoadjuvant treatment for NSCLC, including local radiotherapy, chemotherapy only, and targeted therapy; and (III) patients whose operations included pulmonary arterioplasty and/or bronchoplasty.

\section{Histologic and staging evaluation}

Tissue diagnosis and histologic characterization was performed by percutaneous needle biopsy or endobronchial ultrasound-transbronchial needle aspiration (EBUS-TBNA). Computed tomography (CT) scans were performed to evaluate primary tumors. Positron emission tomography (PET), brain magnetic resonance imaging (MRI), and EBUS-TBNA were performed to confirm the clinical stage.

\section{Neoadjuvant therapy strategy}

Platinum-based doublet chemotherapy combined with PD-1 
inhibitors were prescribed every 21 days for 2-4 treatment cycles depending upon treatment response. The therapeutic regimens of chemotherapy drugs and PD-1 inhibitors are shown in Table 1. According to the protocol, if serious drug-related adverse effects occurred, the use of PD-1 inhibitors were ceased temporarily or permanently. After 2 cycles of neoadjuvant therapy, CT scans or PET-CTs were performed for evaluation of response. If complete resection was not felt to be feasible at that time, additional cycles were considered.

\section{Surgical resection}

The surgical approaches, video-assisted thoracoscopic surgery (VATS) or thoracotomy, were determined at the discretion of surgeon. Surgery was performed 4-6 weeks after the last cycle of neoadjuvant therapy. Surgical procedures included the complete removal of the primary tumor via anatomic resection and systemic lymph node dissection. Double-sleeve resection was performed when the lesions invaded the central vessels. The anastomoses were performed with Prolene running suture and covered by vascular pedicled intercostal muscles, thymus, or pleura. Generally, 3 chest tubes were placed after operation: 2 were placed at the top and the bottom of the pleural cavity for promoting lung recruitment and fluid drainage, respectively. They were removed when patients were discharged without air leak. In addition, another flexible tube was placed at the anastomotic site to prevent the BPF, which was usually removed 1 week after discharge.

\section{Response evaluation}

Radiologic and pathologic responses were evaluated by specialists at the Department of Radiology and the Department of Pathology, Shanghai Pulmonary Hospital. The radiologic response was determined according to the Response Evaluation Criteria for Solid Tumors version 1.1 (RECIST 1.1) (12). Major pathological response (MPR) was defined as the presence of $10 \%$ or less viable tumor cells in the resected primary tumor, and complete pathological response (CPR) was defined as the tumor without any viable tumor in both the removed primary tumor and dissected lymph nodes (13).

\section{Biomarker analysis}

The formalin-fixed and paraffin-embedded specimens were prepared and provided by the Pathology Department of Shanghai Pulmonary Hospital. Slides were stained with PD-1 antibody, PD-L1 antibody, cluster of differentiation (CD)4 antibody, CD8 antibody, CD20 antibody, T cell immunoglobulin mucin 3 (TIM3) antibody, mouse fork head Box Protein P 3 (FXOP3), lymphocyte activation gene 3 (LAG3) antibody, and $\mathrm{T}$ cell immunoglobulin and ITIM domain (TIGIT) antibody. For scoring the immunohistochemistry (IHC) images, IHC scores were calculated using the proportion of positive cells of the whole cells $(0-100)$ multiplied by the average intensity of the positive staining (negative staining as 0 , weak staining as 1 , moderate staining as 2 , and strong staining as 3 ). Thus, for every sample, the IHC score ranged from 1 to 300. The IHC slides were examined and evaluated independently by 3 experienced pathologists, and the mean IHC score was calculated to reduce the potential difference between different observers.

\section{Statistical analyses}

Continuous variables were presented as means \pm standard deviation and were compared using the Mann-Whitney $U$ test. Categorical variables were presented as frequency and respective percentage and were compared using the Pearson chi-squared test or Fisher exact test, as appropriate. All statistical analyses were performed using $\mathrm{R}$ software (version 4.0.3). A 2-sided $\mathrm{P}$ value less than 0.05 was considered statistically significant.

\section{Results}

\section{Patient characteristics}

Twenty-three patients met inclusion criteria for this study (Figure 1), of whom 2 (8.7\%) patients were from the sintilimab clinical trial (registration number: ChiCTR1900023758), 4 (17.4\%) patients were from the camrelizumab clinical trial (registration number: NCT04379739), and 17 (73.9\%) patients were treated off-trial.

The mean age of included patients was $63.2 \pm 7.0$ years, and $22(95.6 \%)$ patients were male. Disease distribution in stages IIB, IIIA and IIIB consisted of 2 (8.7\%), 14 (60.9\%), and $7(30.4 \%)$ patients, respectively. Histopathological diagnosis from pre-treatment biopsies identified 18 (78.3\%) patients with squamous cell carcinoma, 3 (13.0\%) with adenocarcinoma, and $2(8.7 \%)$ with NSCLC. There were 8 (34.8\%) patients who underwent VATS procedures and 15 


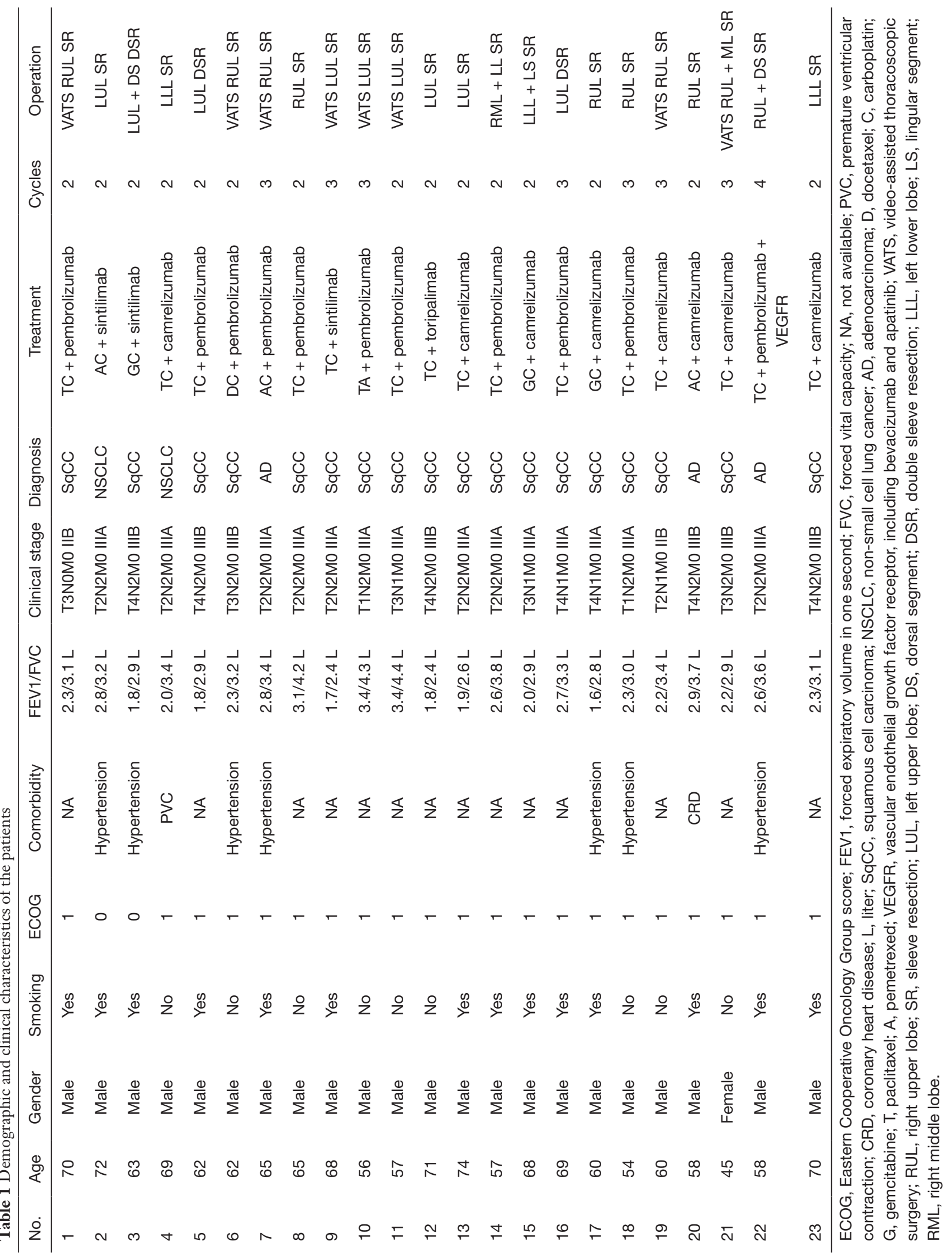




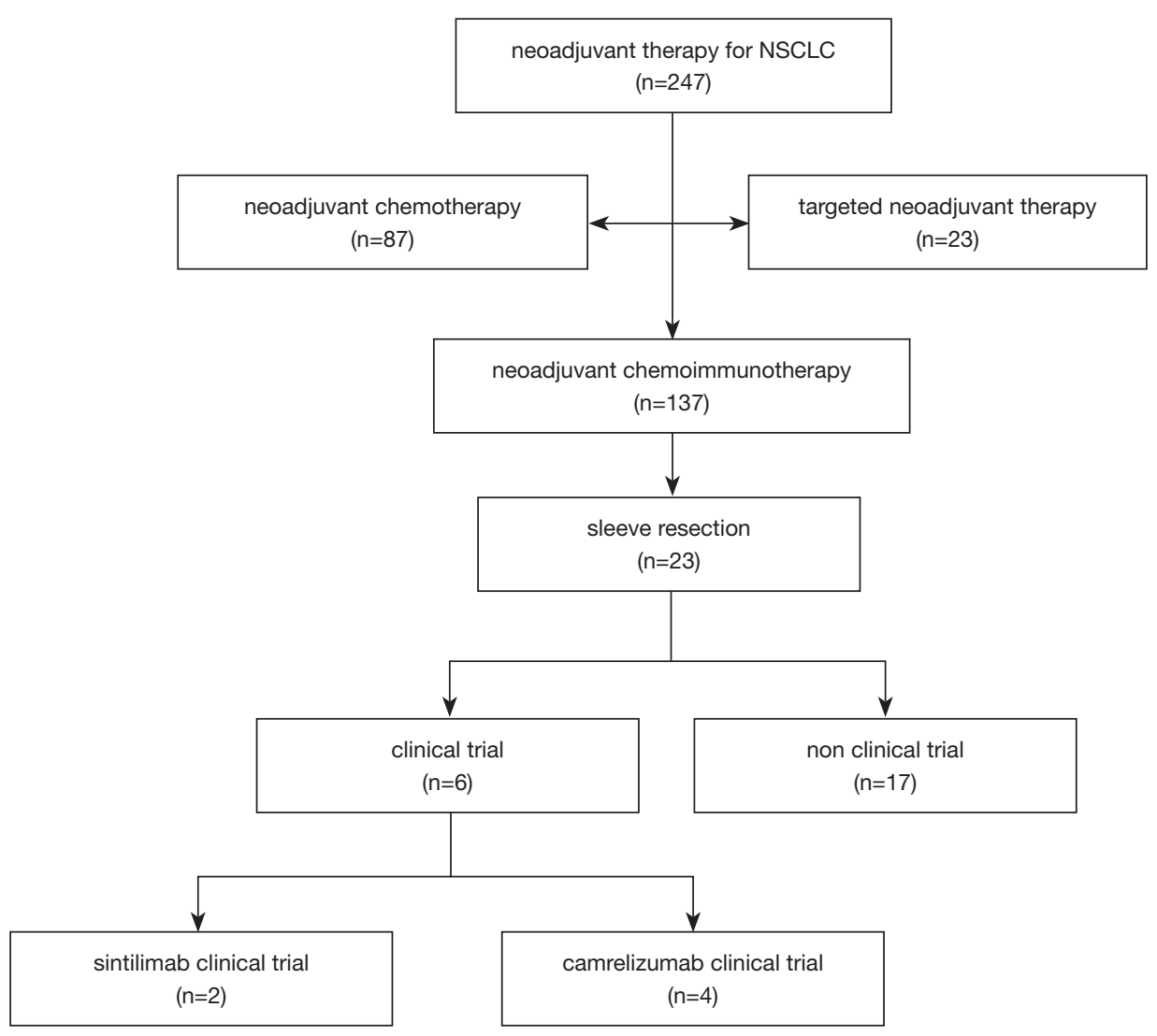

Figure 1 Patient selection flow chart. NSCLC, non-small cell lung cancer.

who underwent thoracotomy (Table 1).

\section{Drug safety}

Treatment-related adverse events (TRAEs) were recorded in $17(73.9 \%)$ patients, but no patients exhibited serious TRAEs. TRAEs of grade $1-2$ occurred in $17(73.9 \%)$ patients, and TRAEs of grade 3-4 occurred in $2(8.7 \%)$ patients. The most common TRAEs were anemia and neutropenia, in $11(47.8 \%)$ patients and 7 (30.4\%) patients, respectively.

\section{Outcomes}

The interval between the end of the last neoadjuvant therapy cycle and surgery was $31.5 \pm 8.9$ days. The mean operative time was $208.1 \pm 72.0 \mathrm{~min}$, intraoperative blood loss was $125.2 \pm 160.0 \mathrm{~mL}$, postoperative hospitalization stay was $7.9 \pm 9.2$ days, and chest tube removal time was $9.8 \pm 7.0$ days. There were no statistical differences in operative time
(198.8 879.7 vs. $225.5 \pm 55.0 \mathrm{~min}, \mathrm{P}=0.197$ ), estimated blood loss $(87.5 \pm 51.8$ vs. $193.9 \pm 145.3 \mathrm{~mL}, \mathrm{P}=0.655)$, chest tube removal time $(7.1 \pm 3.4$ vs. $11.3 \pm 8.0$ days, $\mathrm{P}=0.297)$, and hospitalization time $(5.5 \pm 2.8$ vs. $9.2 \pm 11.2$ days, $\mathrm{P}=0.416)$ between the VATS and thoracotomy groups. In the VATS group, one patient was converted to open surgery due to extensive pleural adhesion (Table 2).

Postoperative complications occurred in 3 (13.0\%) patients, including 1 case with BPF on postoperative day 12 , 1 acute coronary syndrome, and 1 with pneumonia. All of these patients were in the thoracotomy group. The patient with BPF underwent sleeve resection of the upper lobe and superior segment of the right lung. BPF was diagnosed on day 12 via bronchoscopy, and fistula repair was attempted but failed due to severe inflammation at the site of anastomosis. The patient died of cardiac arrest on postoperative day 13 (Figure 2). With a median follow-up of 15 months (range, 10-30 months), only one patient (Patient 1) had local recurrence at 12 months after surgery. This patient received radiofrequency ablation and was still alive 
Table 2 Comparison of perioperative outcomes between the VATS and thoracotomy groups

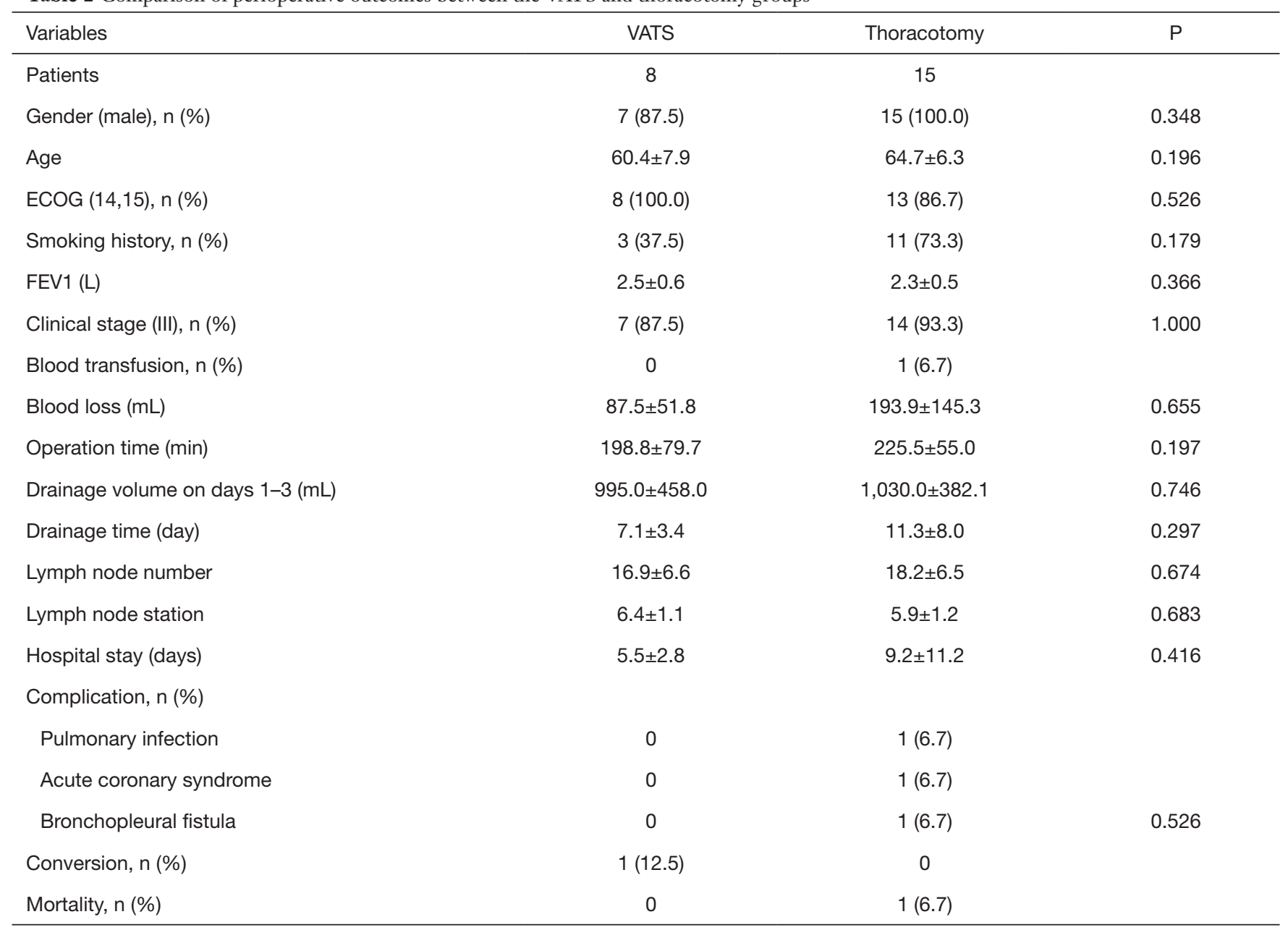

VATS, video-assisted thoracoscopic surgery; ECOG, eastern Cooperative Oncology Group; FEV1, forced expiratory volume in one second; L, liter; mL, milliliter; min, minute.

at the time of data cutoff (Feb 13, 2022).

\section{Efficacy of neoadjuvant therapy}

Preoperative radiological evaluation suggested that there were $5(21.7 \%)$ patients with complete response (CR), 14 (60.9\%) patients with partial response (PR), and 4 (17.4\%) patients with stable disease (SD). There was no progressive disease. A total of $18(78.3 \%)$ patients achieved tumor downstaging. Among them, 16 (69.6\%) patients achieved LN downstaging, including $\mathrm{N} 2$ downstaging to $\mathrm{N} 1$ in 3 (29.2\%) patients and N2 downstaging to N0 in 9 (29.2\%) patients (Figure 3). Postoperative pathological evaluation of the 23 patients revealed that $13(56.5 \%)$ patients achieved MPR, of which seven (27.0\%) patients achieved CPR (Figure 4).

\section{Clinical factors and biomarkers associated with pathological response}

We explored the clinical factors and the IHC score of biomarkers involving antitumor immune response that may be associated with MPR. The patients' demographic characteristics were not significantly different between the MPR and non-MPR groups. Only preoperative histopathologic type and radiological response were statistically associated with MPR. When analyzing the IHC score of the selected biomarkers, the expression of CD4 (16.5 \pm 7.5 vs. $22.3 \pm 17.3)$, CD8 $(21.6 \pm 16.5$ vs. $30.2 \pm 30.5)$, and CD20 $(56.1 \pm 36.8$ vs. $81.5 \pm 49.4$ ) were modestly higher in the MPR group, while the expression of PD-1 (14.6 \pm 18.7 vs. $2.8 \pm 2.9)$, LAG3 $(2.0 \pm 6.3$ vs. 0$)$, and TIGIT $(6.8 \pm 7.9 v s .3 .4 \pm 3.4)$ were lower in the 


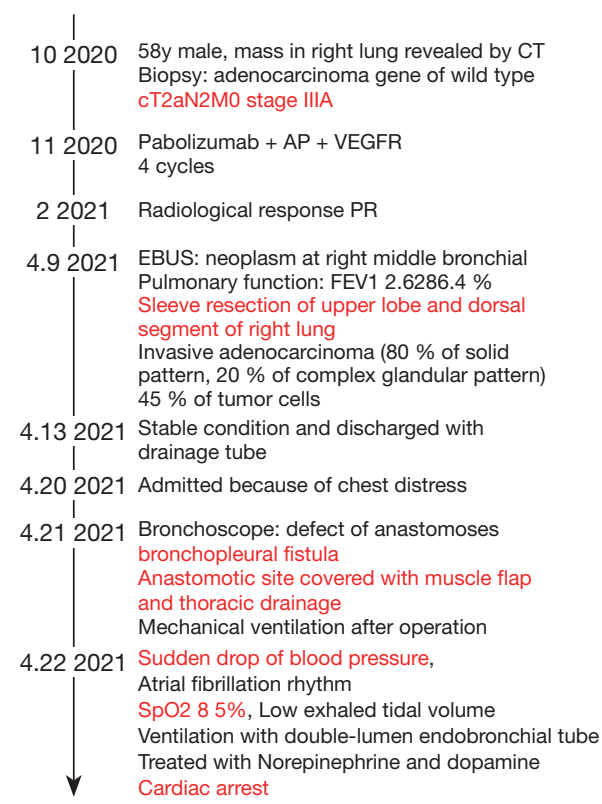

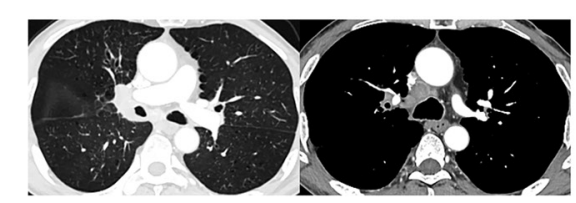

PET (4.22021) increasing metabolism of right upper bronchus, stenosis of the right main bronchus with increasing metabolism, metastasis of mediastinal and right hilar lymph nodes.
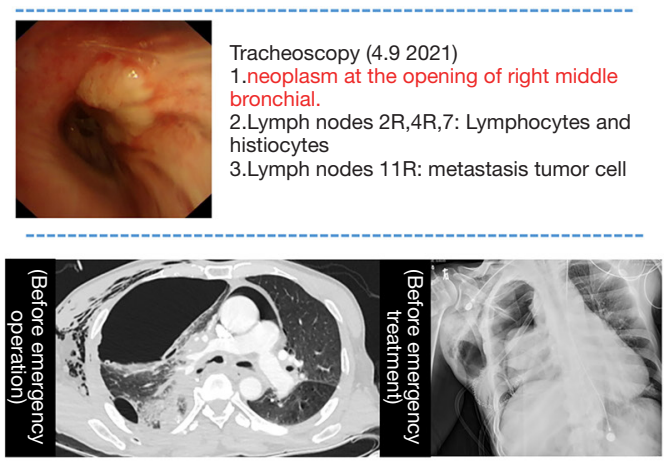

Figure 2 The treatment process of patients complicated with bronchopleural fistula. CT, computed tomography; AP, pemetrexed and cisplatin; VEGFR, vascular endothelial growth factor receptor; PR, partial response; PET, positron emission tomography; EBUS, endobronchial ultrasound; FEV1, forced expiratory volume in one second.

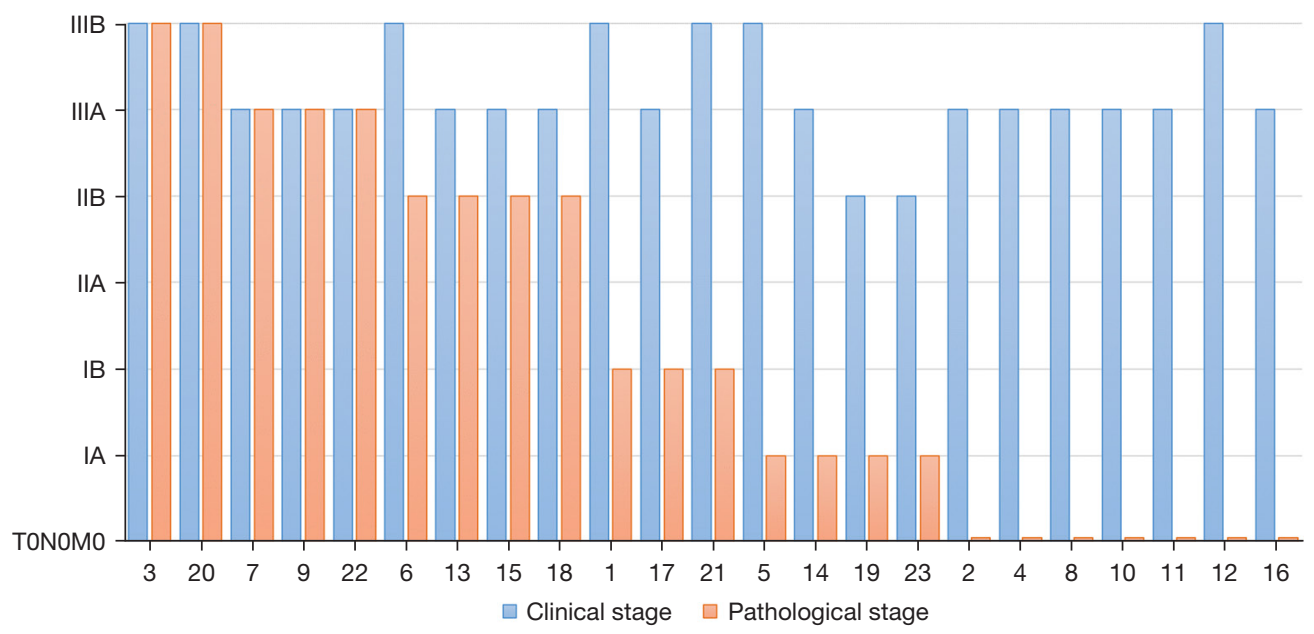

Figure 3 Stages of patients before and after neoadjuvant therapy. $x$-axis presented the patient number, and y-axis presented the clinical stage.

MPR group compared to the non-MPR group, although the difference was not significant (Table 3).

\section{Discussion}

In the present study, we report the results of sleeve resection after neoadjuvant chemoimmunotherapy in locally advanced NSCLC. Our results showed that TRAEs occurred in 17
(73.9\%) patients, and no serious TRAE was recorded from the drugs received. All patients achieved R0 resection. Postoperative complications were observed in 3 patients, with no difference between VATS and thoracotomy. One serious complication occurred, death of BPF, which has led to lessons learned in terms of patient selection and technical caveats. Nonetheless, the objective response rate was $82.6 \%$, and MPR and PCR were achieved in 13 (56.5\%) 


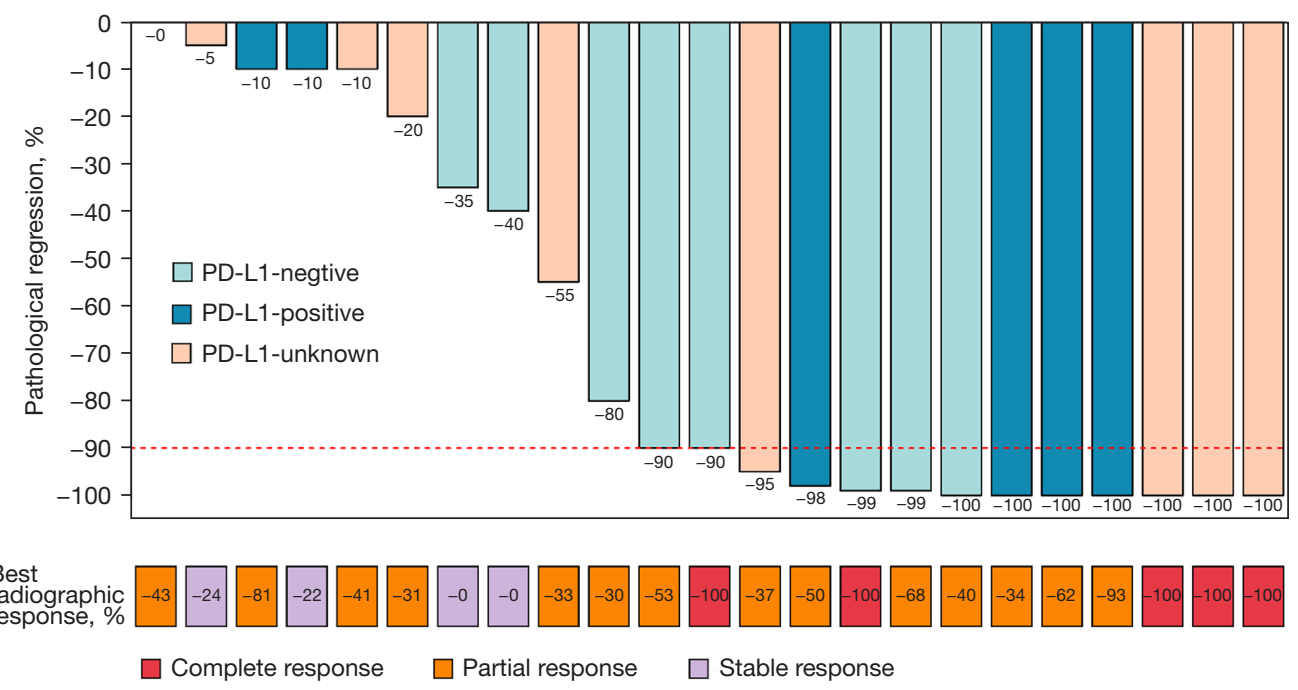

Figure 4 The correlation between radiological response and pathological response. PD-L1, programmed cell death ligand 1.

and $7(27.0 \%)$ patients, respectively.

The perioperative outcomes of sleeve resection after neoadjuvant chemoimmunotherapy have remained unclear due to the lack of prior relevant studies. Chen et al.'s study involving 9 patients showed no difference in surgical time and number of lymph nodes between sleeve resection after neoadjuvant chemoimmunotherapy and upfront surgery (16). Our study, representing the largest reported cohort thus far, found that sleeve resection after the neoadjuvant chemoimmunotherapy exhibited similar perioperative outcomes compared with sleeve resection alone (17). In addition, postoperative complications occurred in $3(13.0 \%)$ patients in the thoracotomy group, of which $1(4.3 \%)$ patient died of BPF. Specifically, the patient with BPF received right upper sleeve lobectomy plus S6 segmentectomy, and no bronchial stump coverage was used during operation. It is possible that right upper lobectomy plus S6 segmentectomy can result in a large residual cavity in the right upper thorax. The fluid might be easily accumulated in the anastomotic site, thus leading to an increased risk of BPF. Therefore, adequate drainage and bronchial stump reinforcement were important for patients with a high-risk of BPF. Overall, the introduction of neoadjuvant immunotherapy did not appear to increase the risks of postoperative complications compared to neoadjuvant chemotherapy alone (18). A dominant concern after neoadjuvant immunotherapy was that more anastomosis-related complications might occur, yet the incidence of BPF in this study was comparable to previous literature (19). Therefore, sleeve resection after neoadjuvant immunochemotherapy appeared to show promising perioperative results without increased surgical complications.

In previous studies, most operations after neoadjuvant chemoimmunotherapy were performed via thoracotomy due to concern about the potential risks of intraoperative crisis $(14,15)$, and thus, the use of VATS has been slow to achieve adoption. Bott et al. reported that 7 (54\%) patients undergoing a minimally invasive approach required conversion to open surgery, and postoperative morbidity occurred in 10 of 20 (50\%) patients (20). Liang et al. also reported a high proportion of conversion after neoadjuvant chemoimmunotherapy due to pleural adhesions (10). In this study, only one patient in the VATS group was converted to thoracotomy due to extensive pleural adhesion, who was in grade 4 (i.e., complete symphysis of the pleural surfaces) in the pleural adhesion grading system documented by Mason et al. (21). VATS did not prolong the operative time or increase the risks of intraoperative blood loss compared with thoracotomy. These results demonstrated the feasibility of VATS in the treatment of locally advanced NSCLC after neoadjuvant chemoimmunotherapy, and patients without extensive pleural adhesion, huge metastasized lymph nodes, and severe invasion to the hilum were found to be the optimal candidates for VATS.

The promising pathological response of neoadjuvant PD-1 inhibitors were hopeful to change the therapeutic regimes of NSCLC. The NADIM clinical trial firstly assessed the effectiveness and safety of neoadjuvant 
Table 3 Comparison of the clinical characteristics and IHC scores between the MPR and non-MPR groups

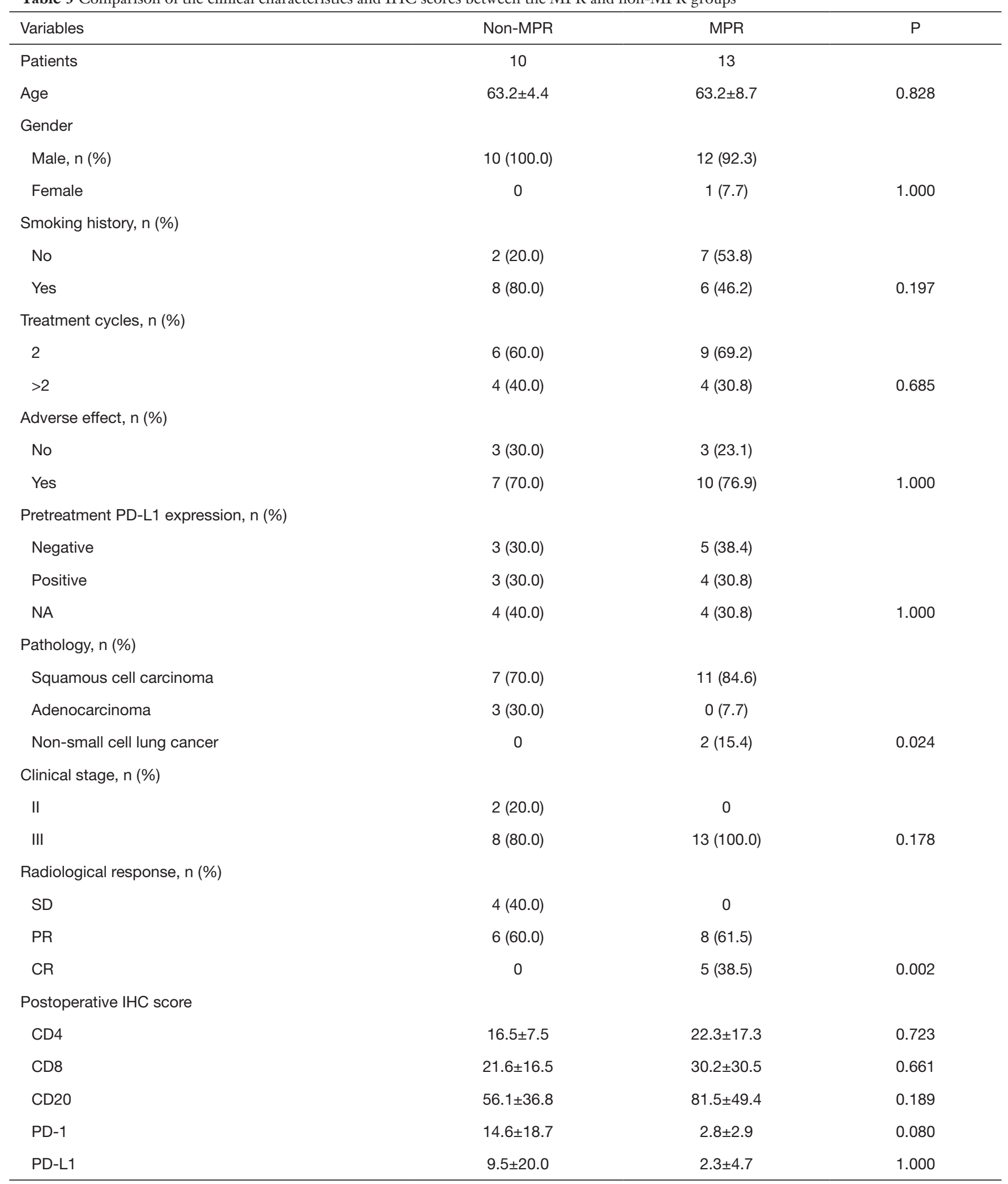

Table 3 (continued) 
Table 3 (continued)

\begin{tabular}{lccc}
\hline Variables & Non-MPR & MPR & P \\
\hline TIM3 & $0.6 \pm 1.3$ & $3.5 \pm 8.5$ & 0.925 \\
FXOP3 & $3.5 \pm 4.5$ & $3.9 \pm 8.0$ & 0.563 \\
LAG3 & $2.0 \pm 6.3$ & 0 & 0.146 \\
TIGIT & $6.8 \pm 7.9$ & $3.4 \pm 3.4$ & 0.552 \\
\hline
\end{tabular}

IHC, immunohistochemistry; MPR, major pathologic response; PD-L1, programmed cell death ligand 1; NA, not available; SD, stable disease; PR, partial response; CR, complete response; CD, cluster of differentiation; PD-1, the programmed cell death receptor 1; TIM3, T cell immunoglobulin mucin 3; FXOP3, mouse fork head Box Protein P 3; LAG3, lymphocyte-activation gene 3; TIGIT, T cell immunoglobulin and ITIM domain.

chemoimmunotherapy for NSCLC; they found that $34(83 \%)$ patients had MPR and $26(63 \%)$ patients had CPR (22). Moreover, the clinical trial of neoadjuvant atezolizumab reported that 17 of $30(57 \%)$ patients had an MPR (23). Our results also showed a high MPR (56.5\%) after neoadjuvant chemoimmunotherapy. Therefore, neoadjuvant chemoimmunotherapy followed by sleeve resection could potentially improve the therapeutic efficacy and augment the therapeutic strategies offered in the treatment of locally advanced NSCLC.

At present, biomarkers for immunotherapy are investigational. We found that postoperative histopathologic type and radiological response were different between the MPR and non-MPR groups. A similar association between pathologic type and MPR was also observed in another clinical trial, where MPR in squamous cell carcinoma was higher than that in adenocarcinoma (23). This association was possibly due to greater CD8 lymphocyte infiltration in squamous cell carcinoma (24).

PD-L1 expression was widely established as a significant predictor in immunotherapy, and higher PD-L1 expression was predictive of better radiological response as well as longer overall survival (25). While for neoadjuvant chemoimmunotherapy, PD-L1 expression in the tumor cells was not associated with MPR, which were also indicated in our study (22). Although the IHC scores were not significantly different, the expression of CD4, CD8, and CD20 was modestly higher in the MPR group compared with the non-MPR patients (Figure 5). The combination of chemotherapy and immunotherapy had the potential advantage of generating more abundant and diverse neoantigens to enhance systemic immune response, thus recruiting more lymphocytes into the tumor microenvironment and eliminating the micro-metastasis (26).

TIM3 was originally identified as a specific marker for
T helper cells and CD8 T cells, with evidence for TIM3dependent inhibitory function (27). Herein, we found that TIM3 expression was higher in MPR, as the tumor microenvironment enriched more $\mathrm{T}$ cells with a high pathological response. It has been previously reported that PD-1 inhibitors could mediate endocytosis of PD-1 in T cells (28); similarly, we found the expression of PD-1 was lower in MPR, while the expression of PD-L1 was comparable between two groups. We also found that the expression of LAG3 and TIGIT was higher in the non-MPR group; perhaps, this may be due to the activation of other immunosuppressive pathways in non-MPR patients (29).

Several limitations should be considered in gleaning conclusions from this study. Firstly, this was a retrospective review with potential selection bias. The selection of candidates for neoadjuvant therapy and the use of VATS versus thoracotomy were generally made by the discretion of each surgeon. Furthermore, the included population was relatively small and lacked long-term outcomes, which precluded us from analyzing the predictive characteristics and biomarkers of postoperative complications and MPR, as well as the role of sleeve resection after chemoimmunotherapy on survival.

However, despite these limitations, this represents the largest study to date demonstrating safety and efficacy of the promising role of neoadjuvant chemoimmunotherapy prior to sleeve resection for advanced NSCLC.

\section{Conclusions}

In conclusion, we reported the perioperative outcomes of sleeve resection after neoadjuvant chemoimmunotherapy in the treatment of NSCLC. Sleeve resection after neoadjuvant chemoimmunotherapy was feasible and effective in patients with locally advanced NSCLC, with comparable 


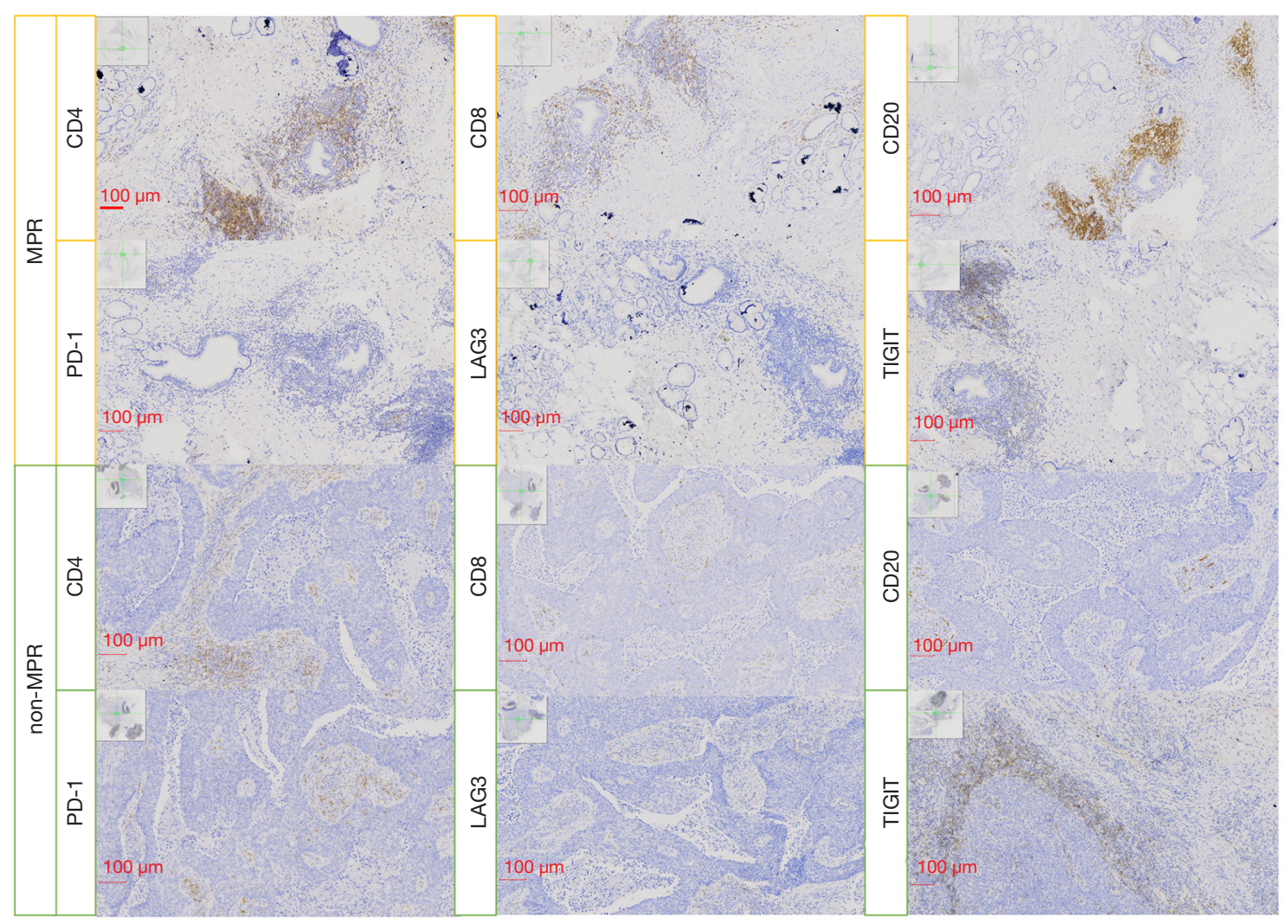

Figure 5 The IHC score of biomarkers involved in the immune response in the MPR and non-MPR groups (scale bar, $100 \mu \mathrm{m}$ ). IHC, immunohistochemistry; MPR, major pathological response.

perioperative outcomes between VATS and thoracotomy, and complete resection could be achieved in all patients. Further prospective studies with larger patient sample sizes and longterm follow-up are needed to consolidate these findings.

\section{Acknowledgments}

The authors appreciate the academic support from the AME Thoracic Surgery Collaborative Group.

Funding: This study was supported by the National Natural Science Foundation of China (Grant No. 82172848), the Shanghai Rising-Star Program (Grant No. 19QA1407400), the Shanghai Municipal Education Commission "Chen Guang" project (19CG19), the Shanghai "Rising Stars of Medical Talent" Youth Development Program Youth Medical Talents-Specialist Program, and Shanghai Pulmonary Hospital Fund (Nos. fkyq1908 and fkzr2105).

\section{Footnote}

Reporting Checklist: The authors have completed the STROBE reporting checklist. Available at https://tlcr. amegroups.com/article/view/10.21037/tlcr-22-56/rc

Data Sharing Statement: Available at https://tlcr.amegroups. com/article/view/10.21037/tlcr-22-56/dss

Conflicts of Interest: All authors have completed the ICMJE uniform disclosure form (available at https://tlcr.amegroups. com/article/view/10.21037/tlcr-22-56/coif). RAR reports grants from Merck; consulting fees from Astra-Zeneca, Novartis, EMD Serono, and Amgen; payment from AstraZeneca, Genentech, and Merck. The other authors have no conflicts of interest to declare.

Ethical Statement: The authors are accountable for all aspects 
of the work in ensuring that questions related to the accuracy or integrity of any part of the work are appropriately investigated and resolved. This study protocol was approved by the Ethics Committee of Shanghai Pulmonary Hospital (No. 19216XW-4), and the study protocols conformed to the provisions of the Declaration of Helsinki (as revised in 2013). For its retrospective nature, written consents from the included patients were not needed.

Open Access Statement: This is an Open Access article distributed in accordance with the Creative Commons AttributionNonCommercial-NoDerivs 4.0 International License (CC BYNC-ND 4.0), which permits the non-commercial replication and distribution of the article with the strict proviso that no changes or edits are made and the original work is properly cited (including links to both the formal publication through the relevant DOI and the license). See: https://creativecommons. org/licenses/by-nc-nd/4.0/.

\section{References}

1. Chansky K, Detterbeck FC, Nicholson AG, et al. The IASLC Lung Cancer Staging Project: External Validation of the Revision of the TNM Stage Groupings in the Eighth Edition of the TNM Classification of Lung Cancer. J Thorac Oncol 2017;12:1109-21.

2. Migliorino MR, De Petris L, De Santis S, et al. Locally advanced non-small cell lung cancer: role of induction chemotherapy in resectable N2 disease. Ann Oncol 2006;17 Suppl 2:ii28-31.

3. Bogart JA, Aronowitz JN. Localized non-small cell lung cancer: adjuvant radiotherapy in the era of effective systemic therapy. Clin Cancer Res 2005;11:5004s-10s.

4. NSCLC Meta-analysis Collaborative Group. Preoperative chemotherapy for non-small-cell lung cancer: a systematic review and meta-analysis of individual participant data. Lancet 2014;383:1561-71.

5. Pignon JP, Tribodet H, Scagliotti GV, et al. Lung adjuvant cisplatin evaluation: a pooled analysis by the LACE Collaborative Group. J Clin Oncol 2008;26:3552-9.

6. Gong J, Chehrazi-Raffle A, Reddi S, et al. Development of PD-1 and PD-L1 inhibitors as a form of cancer immunotherapy: a comprehensive review of registration trials and future considerations. J Immunother Cancer 2018;6:8.

7. Forde PM, Chaft JE, Smith KN, et al. Neoadjuvant PD-1 Blockade in Resectable Lung Cancer. N Engl J Med 2018;378:1976-86.
8. Felip E, Altorki N, Zhou C, et al. Adjuvant atezolizumab after adjuvant chemotherapy in resected stage IBIIIA non-small-cell lung cancer (IMpower010): a randomised, multicentre, open-label, phase 3 trial. Lancet 2021;398:1344-57.

9. Cusumano $\mathrm{G}$, Marra $\mathrm{A}$, Lococo $\mathrm{F}$, et al. Is sleeve lobectomy comparable in terms of short- and long-term results with pneumonectomy after induction therapy? A multicenter analysis. Ann Thorac Surg 2014;98:975-83.

10. Liang H, Yang C, Gonzalez-Rivas D, et al. Sleeve lobectomy after neoadjuvant chemoimmunotherapy/ chemotherapy for local advanced non-small cell lung cancer. Transl Lung Cancer Res 2021;10:143-55.

11. Rendina EA, Venuta F, De Giacomo T, et al. Safety and efficacy of bronchovascular reconstruction after induction chemotherapy for lung cancer. J Thorac Cardiovasc Surg 1997;114:830-5; discussion 835-7.

12. Eisenhauer EA, Therasse P, Bogaerts J, et al. New response evaluation criteria in solid tumours: revised RECIST guideline (version 1.1). Eur J Cancer 2009;45:228-47.

13. Mouillet G, Monnet E, Milleron B, et al. Pathologic complete response to preoperative chemotherapy predicts cure in early-stage non-small-cell lung cancer: combined analysis of two IFCT randomized trials. J Thorac Oncol 2012;7:841-9.

14. Bott MJ, Cools-Lartigue J, Tan KS, et al. Safety and Feasibility of Lung Resection After Immunotherapy for Metastatic or Unresectable Tumors. Ann Thorac Surg 2018;106:178-83.

15. Chaft JE, Hellmann MD, Velez MJ, et al. Initial Experience With Lung Cancer Resection After Treatment With T-Cell Checkpoint Inhibitors. Ann Thorac Surg 2017;104:e217-8.

16. Chen Y, Zhang L, Yan B, et al. Feasibility of sleeve lobectomy after neo-adjuvant chemo-immunotherapy in non-small cell lung cancer. Transl Lung Cancer Res 2020;9:761-7.

17. Pagès PB, Mordant $P$, Renaud $S$, et al. Sleeve lobectomy may provide better outcomes than pneumonectomy for non-small cell lung cancer. A decade in a nationwide study. J Thorac Cardiovasc Surg 2017;153:184-195.e3.

18. Inci I, Benker M, Çitak N, et al. Complex sleeve lobectomy has the same surgical outcome when compared with conventional lobectomy in patients with lung cancer. Eur J Cardiothorac Surg 2020;57:860-6.

19. Milman S, Kim AW, Warren WH, et al. The incidence of perioperative anastomotic complications after sleeve lobectomy is not increased after neoadjuvant chemoradiotherapy. Ann Thorac Surg 2009;88:945-50; 
discussion 950-1.

20. Bott MJ, Yang SC, Park BJ, et al. Initial results of pulmonary resection after neoadjuvant nivolumab in patients with resectable non-small cell lung cancer. J Thorac Cardiovasc Surg 2019;158:269-76.

21. Mason AC, Miller BH, Krasna MJ, et al. Accuracy of CT for the detection of pleural adhesions: correlation with video-assisted thoracoscopic surgery. Chest 1999;115:423-7.

22. Provencio M, Nadal E, Insa A, et al. Neoadjuvant chemotherapy and nivolumab in resectable non-small-cell lung cancer (NADIM): an open-label, multicentre, singlearm, phase 2 trial. Lancet Oncol 2020;21:1413-22.

23. Shu CA, Gainor JF, Awad MM, et al. Neoadjuvant atezolizumab and chemotherapy in patients with resectable non-small-cell lung cancer: an open-label, multicentre, single-arm, phase 2 trial. Lancet Oncol 2020;21:786-95.

24. Meng X, Gao Y, Yang L, et al. Immune Microenvironment Differences Between Squamous and Non-squamous Non-small-cell Lung Cancer and Their Influence on the Prognosis. Clin Lung Cancer 2019;20:48-58.

Cite this article as: Dai J, Zhu X, Li D, Huang Y, Liu X, He W, Duan L, Zhao D, Zhu Y, Chen C, Provencio M, Ramirez RA, Antonoff MB, Wu C, Jiang G. Sleeve resection after neoadjuvant chemoimmunotherapy in the treatment of locally advanced non-small cell lung cancer. Transl Lung Cancer Res 2022;11(2):188-200. doi: 10.21037/tlcr-22-56
25. Sezer A, Kilickap S, Gümüş M, et al. Cemiplimab monotherapy for first-line treatment of advanced nonsmall-cell lung cancer with PD-L1 of at least $50 \%$ : a multicentre, open-label, global, phase 3, randomised, controlled trial. Lancet 202 1;397:592-604.

26. Liu J, Blake SJ, Yong MC, et al. Improved Efficacy of Neoadjuvant Compared to Adjuvant Immunotherapy to Eradicate Metastatic Disease. Cancer Discov 2016;6:1382-99.

27. Meyers JH, Sabatos CA, Chakravarti S, et al. The TIM gene family regulates autoimmune and allergic diseases. Trends Mol Med 2005;11:362-9.

28. Burr ML, Sparbier CE, Chan YC, et al. CMTM6 maintains the expression of $\mathrm{PD}-\mathrm{L} 1$ and regulates antitumour immunity. Nature 2017;549:101-5.

29. Anderson AC, Joller N, Kuchroo VK. Lag-3, Tim-3, and TIGIT: Co-inhibitory Receptors with Specialized Functions in Immune Regulation. Immunity 2016;44:989-1004.

(English Language Editor: A. Kassem) 